\title{
A recent tipping point in the Arctic sea-ice cover: abrupt and persistent increase in the seasonal cycle since 2007
}

\author{
V. N. Livina ${ }^{1,2}$ and T. M. Lenton ${ }^{3}$ \\ ${ }^{1}$ National Physical Laboratory, Teddington, Middlesex TW11 0LW, UK \\ ${ }^{2}$ School of Environmental Sciences, University of East Anglia, Norwich NR4 7TJ, UK \\ ${ }^{3}$ College of Life and Environmental Sciences, University of Exeter, Hatherly Laboratories, Exeter EX4 4PS, UK
}

Correspondence to: V. N. Livina (valerie.livina@npl.co.uk)

Received: 26 June 2012 - Published in The Cryosphere Discuss.: 18 July 2012

Revised: 7 January 2013 - Accepted: 18 January 2013 - Published: 12 February 2013

\begin{abstract}
There is ongoing debate over whether Arctic sea ice has already passed a "tipping point", or whether it will do so in the future. Several recent studies argue that the loss of summer sea ice does not involve an irreversible bifurcation, because it is highly reversible in models. However, a broader definition of a "tipping point" also includes other abrupt, non-linear changes that are neither bifurcations nor necessarily irreversible. Examination of satellite data for Arctic sea-ice area reveals an abrupt increase in the amplitude of seasonal variability in 2007 that has persisted since then. We identified this abrupt transition using recently developed methods that can detect multi-modality in time-series data and sometimes forewarn of bifurcations. When removing the mean seasonal cycle (up to 2008) from the satellite data, the residual sea-ice fluctuations switch from uni-modal to multimodal behaviour around 2007. We originally interpreted this as a bifurcation in which a new lower ice cover attractor appears in deseasonalised fluctuations and is sampled in every summer-autumn from 2007 onwards. However, this interpretation is clearly sensitive to how the seasonal cycle is removed from the raw data, and to the presence of continental land masses restricting winter-spring ice fluctuations. Furthermore, there was no robust early warning signal of critical slowing down prior to the hypothesized bifurcation. Early warning indicators do however show destabilization of the summer-autumn sea-ice cover since 2007. Thus, the bifurcation hypothesis lacks consistent support, but there was an abrupt and persistent increase in the amplitude of the seasonal cycle of Arctic sea-ice cover in 2007, which we describe as a (non-bifurcation) "tipping point". Our statistical methods detect this "tipping point" and its time of onset. We
\end{abstract}

discuss potential geophysical mechanisms behind it, which should be the subject of further work with process-based models.

\section{Introduction}

Arctic sea ice has experienced striking reductions in areal coverage (Stroeve et al., 2007; Nghiem et al., 2007), especially in recent summers, with 2007-2012 having the six lowest ice cover minima in the satellite record (Fig. 1). Observations have fallen below IPCC model projections (Stroeve et al., 2007), despite the models having been in agreement with the observations in the 1970s. The latest models are more consistent with satellite observations (1979-present), but still fail to capture the full extent of the observed downward trend (Stroeve et al., 2012). Summer ice cover is forecast to disappear later this century (Boe et al., 2009), but the nature of the underlying transition is debated (Lenton et al., 2008; Lindsay and Zhang, 2005; Amstrup et al., 2010; Winton, 2006; Eisenman and Wettlaufer, 2009; Tietsche et al., 2011).

Arctic sea ice has been identified as a potential tipping element in the Earth's climate system (Lenton et al., 2008), and at least one study suggests it has already passed a "tipping point" (Lindsay and Zhang, 2005). For the future, some models forecast abrupt ice loss events (Amstrup et al., 2010), on the way to a seasonally ice-free Arctic. These may qualify as passing tipping points following the broad definition given in Lenton et al. (2008) of a point at which a small change in forcing leads to a qualitative change in the future state 

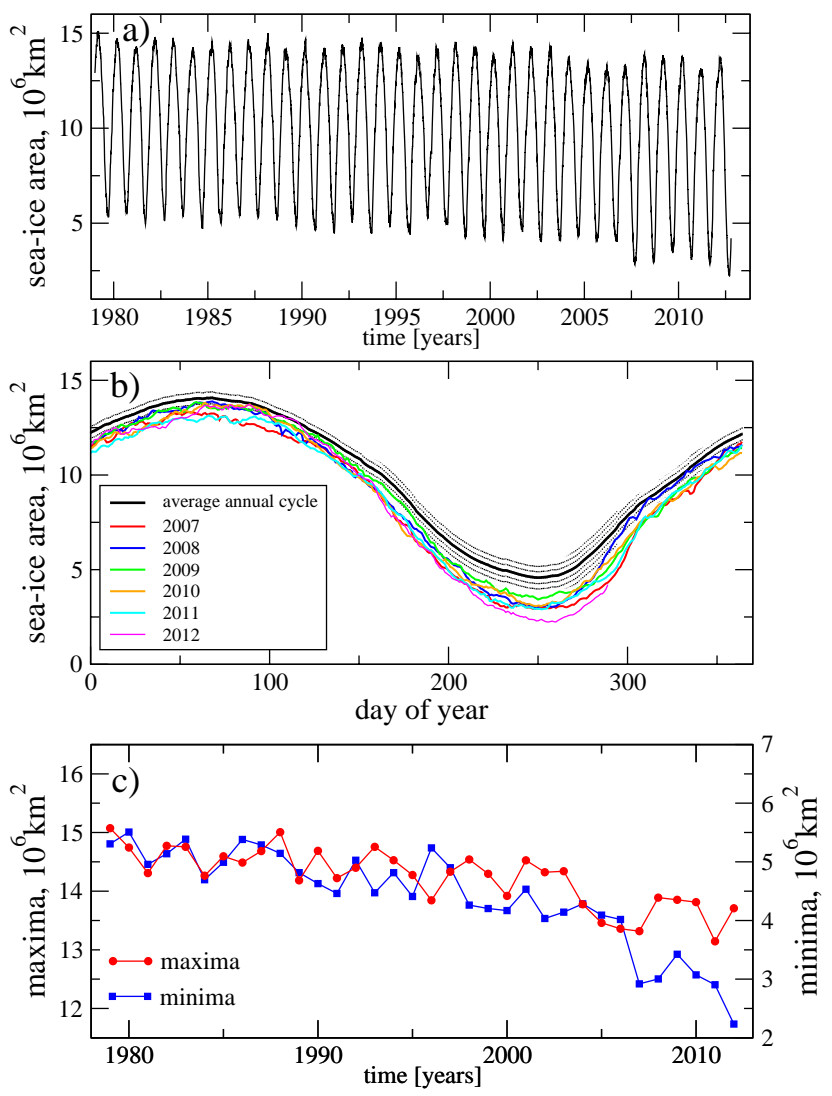

Fig. 1. Arctic sea-ice area from satellite data. (a) Arctic sea-ice area, 1979-2012. (b) The mean annual cycle of the area data over 1979-2008 inclusive (solid line, shaded area denotes two error bars), together with the last five anomalous years. (c) Annual maxima (left axis) and minima (right axis) showing an abrupt increase in amplitude of the seasonal cycle in 2007.

of a system. The definition includes both reversible and irreversible transitions, bifurcations and some non-bifurcation phenomena.

However, most recent papers on the Arctic sea ice opt for a narrower definition of a tipping point, addressing whether summer sea-ice loss will involve an irreversible (e.g. saddlenode/fold) bifurcation. They find instead that in models the loss of summer sea-ice cover is highly reversible (Amstrup et al., 2010; Winton, 2006; Eisenman and Wettlaufer, 2009; Tietsche et al., 2011). Abrupt ice loss events are then attributed to the loss of year-round sea ice in the Arctic making the remaining ice more vulnerable to summer melt, and prone to larger fluctuations in area coverage (Notz et al., 2009). An exception is a recent model (Abbot et al., 2011) showing that positive feedbacks involving clouds can create multiple stable states for seasonal ice cover and bifurcations between them. Furthermore, models of past abrupt climate changes in the Arctic have shown multiple stable states for sea-ice cover in the Barents and Kara seas region and abrupt switches between them (Bengtsson et al., 2004; Semenov et al., 2009).
This suggests that sub-Arctic-scale "tipping points" in seaice cover are conceivable.

On viewing the satellite-derived daily record of sea-ice area from 1979 to present (Fig. 1a), it is clear that the last six years have been characterized by an increase in the amplitude of seasonal sea-ice variation (Fig. 1b). The annual ice cover minimum dropped an order of $\sim 10^{6} \mathrm{~km}^{2}$ more than the annual maximum in 2007, and the difference has been maintained since then (Fig. 1c). This already suggests an abrupt and persistent change in sea-ice dynamics. It led us to hypothesize that the sea ice may have passed a bifurcation-type tipping point, in which a new attractor for lower summer-autumn sea-ice cover became stable and began to be sampled in summer 2007, and in every summer since, with seasonal switches to/from the pre-existing attractor - see Fig. 10 of Livina and Lenton (2012).

We arrived at this hypothesis by applying recently developed methods of time-series analysis that can detect changes in the modality of data (Livina et al., 2010, 2011; Cimatoribus et al., 2012) and in some cases forewarn of bifurcations (Scheffer et al., 2009; Held and Kleinen, 2004; Livina and Lenton, 2007; Lenton, 2011). Our analysis concentrates on the satellite-derived daily record of sea-ice area from 1979 to 2011 (Fig. 1a), and is repeated on the shorter record of sea-ice extent from 1979-2009 (Eisenman, 2010) (Fig. A1) in the Appendix. For much of our analysis, a mean seasonal cycle (Fig. 1b) averaged over the period 1979-2008 was removed from the data, because there is a very strong seasonally forced variation in sea-ice area. The averaged seasonal cycle of the Arctic sea ice from 1979-2008 (Fig. 1b) is very close to a sine wave (no asymmetry over seasons), and we were interested in studying the behaviour of fluctuations from this typical state of seasonal variation.

However, our interpretation in terms of a changing number of sea-ice attractors (Livina and Lenton, 2012) can be profoundly altered by changing the interval that is considered the baseline state for the sea ice (Ditlevsen, 2012). The central problem is that any residual seasonal cycle remaining in the data appears bi-modal, and if there was a jump in the amplitude of the seasonal cycle around 2007, it is not possible to remove one average seasonal cycle from the whole record and get rid of all the residual seasonality (Ditlevsen, 2012). We examine this further here with a stochastic model of growing amplitude in the seasonal cycle, by comparing analysis of the artificial data from this model with that of the observed data.

The paper is organized as follows:

Section 2 details the data pre-processing and the methods applied.

Section 3 presents the results and discusses them, including some tentative geophysical interpretation.

Section 4 concludes. 


\section{Methodology}

\subsection{Data and pre-processing}

Sea-ice area takes into account the fraction of a grid cell that is covered by sea ice, and can be biased low, especially in summer when melt ponds are present. Sea-ice extent assumes that any grid point with more than a certain per cent (for instance, $15 \%$ ) sea ice concentration is totally covered.

Sea ice area data were obtained from "The Cryosphere Today" project of the University of Illinois. This dataset (http://arctic.atmos.uiuc.edu/cryosphere/timeseries.anom.

1979-2008) uses SSM/I and SMMR series satellite products and spans 1979 to present at daily resolution. The most recent data in this series are derived from the Near-RealTime DMSP SSM/I-SSMIS Daily Polar Gridded Sea Ice Concentrations of the National Snow \& Ice Data Centre (NSIDC) (see Maslanik and Stroeve (1999)).

The sea-ice extent time series was derived by Eisenman (2010) (data available at ftp://ftp.agu.org/apend/gl/2010gl043741) on the basis of sea ice concentration using the NASA Team algorithm from Nimbus-7 SMMR (1978-1987), DMSP SSM/I (1987-2009), and DMSP SSMIS (2008-present) satellite passive microwave radiances on a $25 \times 25 \mathrm{~km}$ polar stereographic grid (Cavalieri et al., 1996; Meier et al., 2006; Maslanik and Stroeve, 1999). During periods of instrumental transitions, the overlapping datasets were averaged. Extent was calculated by summing the areas of all grid boxes with at least $15 \%$ ice concentration. Details of the spatial data interpolation are given by Eisenman (2010). The time series spans 1979-2009, and where it has 2-day resolution (when SMMR operated every other day for three months during the record, in October 1978, December 1987 and January 1988), we interpolate to daily resolution to obtain a homogeneous time series.

For both datasets - area and extent - the mean seasonal cycle over the first $30 \mathrm{yr}$ of data (1979-2008) was removed, as on "The Cryosphere Today" website (and widely reproduced elsewhere). We also examined the effect of constructing and removing a different averaging interval (1979-2011), which produces a very similar residual series, just vertically shifted along the y-axis; i.e. the dynamics of the residual fluctuations remained the same. Hence this gives similar results and we do not show it here.

We also analysed a derived index of "equivalent sea-ice extent" (Eisenman, 2010) (available at $\mathrm{ftp} / / / \mathrm{ftp}$.agu.org/apend/g1/2010g1043741), which is based on the latitude of the sea-ice edge where it is free to migrate, converted to an area, assuming there were no continents present.

\subsection{Potential analysis}

To detect any multi-modality in the sea-ice residual data, we use a recently developed (Livina et al., 2010, 2011) and blind-tested (Livina et al., 2012) method of "potential analysis". This assumes that a system is experiencing sufficient short-term stochastic variability (noise) and that it is sampling all of its available states or attractors (given a sufficiently long time window). Then we take advantage of the fact that the stationary probability distribution of the resulting data is directly related to the shape of the underlying potential, which describes the number of underlying attractors and their stability (Livina et al., 2011). Thus, with a sufficiently long time window of data, one can deduce the number of attractors and their relative stability or instability.

The time series are modelled by the following stochastic differential equation:

$\dot{z}(t)=-U^{\prime}(z)+\sigma \eta$,

where $U$ is a polynomial potential of even order and $\eta$ is a Gaussian white noise process of unit variance. Equation (1) has a corresponding Fokker-Planck equation describing the probability density function, and crucially this has a stationary solution that depends only on the underlying potential function and the noise level, $\sigma$;

$p(z) \sim \exp \frac{-2 U(z)}{\sigma^{2}}$.

This allows the underlying potential to be reconstructed from a kernel probability distribution of time-series data (and an estimate of the noise level) as

$U(z)=-\frac{\sigma^{2}}{2} \log p_{\mathrm{d}}(z)$,

where $p_{\mathrm{d}}$ is the empirical probability density of the data.

We detect the order of the polynomial and hence the number of system states following the method in Livina et al. $(2010,2011)$, plotting the results as a function of window length at the end of each sliding window in a colour contour plot (e.g. Fig. 3b). The rate of correct detection depends on sliding window size (Livina et al., 2011): when the window contains more than 400 data points (which in the case of daily sea-ice data corresponds to about $1.1 \mathrm{yr}$ ), the success rate is $80 \%$, even when noise level is up to five times larger than the depth of the potential well; for larger windows it approaches $98 \%$. A test of the method on artificial data, generated from a model system in which the underlying potential bifurcates from one state to two, illustrates correct detection of the number of system attractors (Fig. 2).

Such tests employ Gaussian white noise, whereas sea-ice data are correlated. In the case of correlated data, the probability density under investigation is the same (a probability density function aggregates data without taking into account its temporal organization). However, correlated data are more 

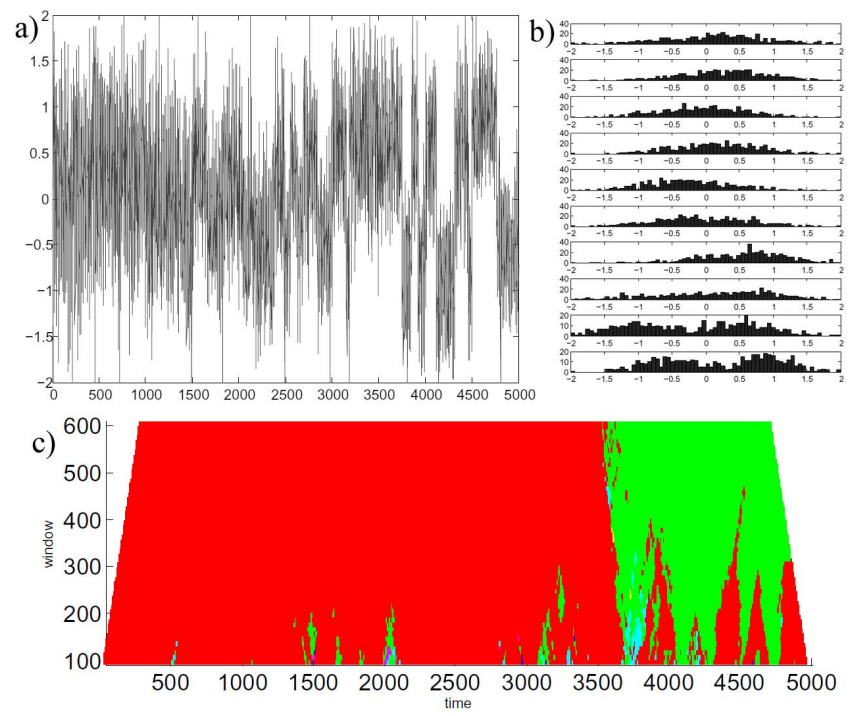

Fig. 2. Test of potential analysis on artificial data from a system bifurcating from one state to two. Here the underlying potential changes smoothly from one-well to double-well, described by the stochastic potential equation with varying potential wells (10 chunks of 500 points each), with the bifurcation occurring at time 3500: (a) artificial data generated from the changing potential function with a noise level 1; (b) histograms of 10 chunks of data, from top to bottom, corresponding to consequent subsets of the series; (c) contour plot of number of detected states, where red $=1$ detected state, green $=2$. Results plotted as a function of sliding window length at the middle of the window.

likely to sample another state due to drift (red noise) than is white noise. Hence correlated data may have better detection rate statistics than uncorrelated data.

We derive the coefficients describing the shape of the potential using an unscented Kalman filter (Livina et al., 2010, 2011), while we estimate the noise level using wavelet denoising with Daubechies wavelets of forth order (Livina et al., 2011).

The method assumes each subset of data is quasistationary and the noise is Gaussian white. For the 4-yr intervals used to reconstruct the potentials (e.g. in Fig. 3c), the assumption of stationarity is reasonable. The noise in geophysical systems may be red rather than white, but the assumption of white noise can still be valid provided that the noise is stationary (detrended fluctuation analysis (DFA) fluctuation exponent less than 1). By applying the potential model in such cases, we may attribute part of the noise variability to the potential dynamics when analysing the two components of the potential model. This model is an approximation; still it allows us to derive accurately the structure of the potential for systems with stationary red noise. When there are no non-stationarities, such noise cannot artificially create an additional system state.

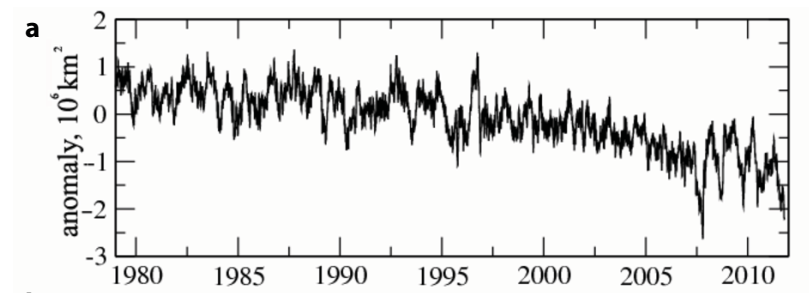

b
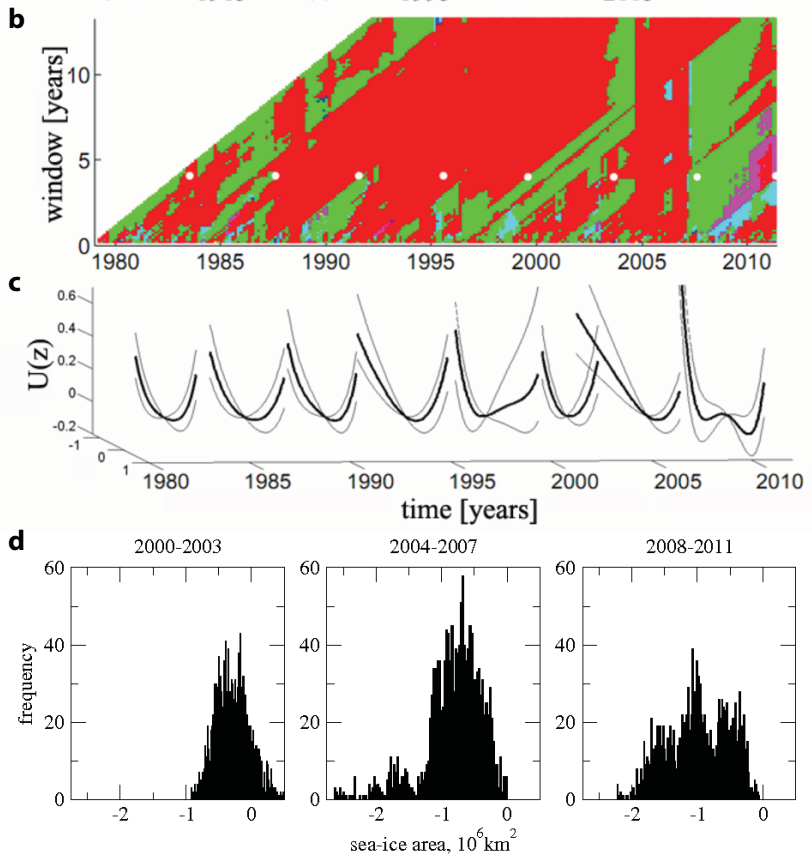

Fig. 3. Analysis of Arctic sea-ice area. (a) Sea-ice area anomaly, daily data with mean seasonal cycle removed. (b) Contour plot of number of detected states, where red $=1$ detected state, green $=2$, cyan $=3$, magenta $=4$. Results plotted as a function of sliding window length at the end of the window. (c) Reconstructed potential curves of eight 4-yr time intervals, corresponding to the white dots in (b). Here $z$ is sea-ice area fluctuation on a shifted scale. Faint lines are potential curves derived from error estimates on the coefficients of the polynomial potential function (for details see Livina et al., 2011). In the penultimate interval 2004-2007, a second state starts to appear and in the final interval 2008-2011 there are two states of comparable stability. (d) Histograms of the data for 20002003, 2004-2007, and 2008-2011 from which the corresponding potential curves are derived (see Methodology).

\subsection{Critical slowing down}

To test for bifurcation in the residual sea-ice fluctuations, we look for the signal of "critical slowing down" beforehand (Scheffer et al., 2009). Namely, for a low-order dynamical system approaching a bifurcation where its current state becomes unstable, and it transitions to some other state, one can expect to see it become more sluggish in its response to small perturbations (Scheffer et al., 2009). This can hold even for complex systems such as the sea ice, if they exhibit a bifurcation point, because near to it their behaviour will reduce down to that of a low-order system (following the center 
manifold theorem). The signal of "critical slowing down" is detectable as increasing autocorrelations in time series data, occurring over timescales capturing the decay of the major mode in the system (Held and Kleinen, 2004), which is controlled by the leading eigenvalue. We looked for this early warning indicator in the form of rising lag-1 autocorrelation function (Held and Kleinen, 2004) (ACF-indicator), and through detrended fluctuation analysis (DFA-indicator) as a rising scaling exponent (Livina and Lenton, 2007). Parabolic trends were removed prior to estimating these two indicators (previously termed "propagators") of critical slowing down. This is because any trend affects autocorrelations and hence may cause false positive signals in the indicators. To test robustness we also performed an alternative pre-processing of data - first removing the quadratic downward trend and then deseasonalising the data, and obtained equivalent results.

\subsubsection{ACF-indicator}

Lag-1 autocorrelation was estimated (Held and Kleinen, 2004; Livina and Lenton, 2007) by fitting an autoregressive model of order 1 (linear AR(1)-process) of the following form:

$z_{t+1}=c \cdot z_{t}+\sigma \eta_{t}$,

where $\eta_{t}$ is a Gaussian white noise process of unit variance, and the "ACF-indicator" (AR1 coefficient) is calculated as

$c=e^{-\kappa \Delta t}$,

where $\kappa$ is the decay rate of perturbations, and $\kappa \rightarrow 0$ (i.e. $c \rightarrow 1$ ) as bifurcation is approached (Held and Kleinen, 2004).

\subsubsection{DFA-indicator}

Detrended fluctuation analysis (DFA) extracts the fluctuation function of window size $s$, which increases as a power law if the data series is long-term power-law correlated:

$F(s) \propto s^{\alpha}$,

where $\alpha$ is the DFA scaling exponent. In the short-term regime, as $c \rightarrow 1$ of the AR(1)-model, the slow exponential decay is well approximated by a power law in which $\alpha \rightarrow 1.5$, in the time interval 10-100 units. Exponent $\alpha$ is rescaled, following Livina and Lenton (2007), to give a "DFA-indicator" that reaches 1 at critical behaviour.

\subsubsection{Variance}

We also monitored variance (calculated as standard deviation), because if a state is becoming less stable this can be characterized by its potential well becoming shallower, causing increased variability over time (although this is not independent of lag-1 autocorrelation; Ditlevsen and Johnsen, 2010).

\subsubsection{Indicator trends}

Upward trends in the indicators (rather than their absolute value) provide the primary early warning signal. The Kendall $\tau$ rank correlation coefficient (Kendall, 1948) measures the strength of the tendency of an indicator to increase (positive values) or decrease (negative values) with time, against the null hypothesis of a random sequence of measurements against time (value approximately zero). As a sensitivity analysis, the sliding window along the time series was varied from $1 / 4$ to $3 / 4$ of the series length.

\subsection{Model of increasing seasonal cycle}

To examine whether the results obtained could be explained by an increase in the amplitude of the seasonal cycle (Ditlevsen, 2012), we built a simple stochastic model, described by the following equation:

$$
\begin{gathered}
x(t)=L+A \cdot \sin \left(\frac{2 \pi t}{365}\right)+\sigma \eta, \\
A=\left\{\begin{array}{l}
1, \text { when } t=1, \ldots, 7300, \\
\frac{0.5}{12410-7300} t+\frac{12410-1.5 \cdot 7300}{12410-7300}, \\
\text { when } t=7301, \ldots, 12410, \operatorname{sign}\left(\sin \left(\frac{2 \pi t}{365}\right)\right)<0,
\end{array}\right.
\end{gathered}
$$

where $\eta$ is Gaussian white noise of unit variance, $\sigma=0.15$. This simulates sinusoidally varying "daily" data (period 365) over a period $t=1: 12410$ corresponding to $34 \mathrm{yr}$, equivalent to period 1979-2012. A global declining trend of the data is simulated as linear in form; $L=-0.02 \cdot t+55.82$. Also the amplitude of the lower half of the sine wave starts to grow linearly after 20 "years" of simulated data (i.e. in "year" 1998), such that it changes from -1 to -1.5 at the end of time series.

The model data were pre-processed similarly to the seaice data; we first deseasonalised the model data (removing 365-day "seasonal" average) and performed potential analysis of the residuals. We then removed a quadratic trend from the series and calculated the early warning indicators.

\section{Results and discussion}

\subsection{Multi-modality detection}

After removing the mean seasonal cycle (1979-2008), the remaining fluctuations in sea-ice area include some of the order of $10^{6} \mathrm{~km}^{2}$ (Fig. 3a). The largest anomalies are in 1996 (maximum of the series) and 2007-2011 (minima). They typically occur in the summer-autumn, when the sea-ice area is at its lowest in the seasonal cycle. Given the size of sea-ice fluctuations during 2007-2011 (Fig. 3a) and the pronounced drop in sea-ice minima relative to sea-ice maxima since 2007 (Fig. 1c), we considered whether the residuals exhibited an abrupt change to multi-modality in 2007.

On analysing the residual sea-ice area fluctuations using our method of potential analysis, over long time windows 
(here $>1 \mathrm{yr}$ ), we typically find a single mode and corresponding attractor, representing the normal seasonal cycle of seaice variability (Fig. 3b). Sometimes a second mode is detected associated with, for example, the sea-ice maximum in 1996, but these changes are not found simultaneously and persistently across a wide range of window lengths. However, from 2007 onwards, a persistent switch to two modes or attractors is detected, across a wide range of window lengths up to $>10 \mathrm{yr}$ (Fig. 3b). The same switch is also detected in analysis of the shorter record of sea-ice extent data (Fig. A2b).

The stability of the attractor(s) for the residual sea-ice fluctuations can be reconstructed, in the form of potential curves for fixed intervals of the data (Fig. 3c), with associated error estimates (on the coefficients of the polynomial function describing the potential; Livina et al., 2011). The seaice residuals are typically characterized by a single mode and corresponding attractor. The interval 1996-1999 (including the 1996 maximum anomaly) shows signs of a second higher ice cover attractor that is degenerate (i.e. not fully stable). In 2000-2003 there is a return to a single attractor. In 2004-2007, which includes the extreme September 2007 sea-ice retreat, a low ice-cover attractor starts to appear in the fluctuations. Then in 2008-2011 the potential separates into two attractors, although the error range allows for one or the other of these to be degenerate.

The potential curves are derived from histograms of the original data (Livina et al., 2011) (Fig. 3d), which confirm a second mode appearing among a long tail of negative fluctuations during 2004-2007, followed by a separation of multiple modes during 2008-2011, which the method fits as a bimodal distribution. Thus, we originally hypothesized that the Arctic sea ice recently passed a bifurcation point (Livina and Lenton, 2012), which created a new lower ice cover attractor for the residual deseasonalised fluctuations. Since then it has fluctuated between its normal attractor for seasonal variability and the new, lower ice cover attractor.

However, an abrupt change in the amplitude of the seasonal cycle will leave a residual record that has some seasonality on one side of the transition or the other (Ditlevsen, 2012). These remnant seasonal fluctuations will in turn produce a bi-modal distribution, which is accurately detected by our method - hence care is needed over how to interpret this. Sure enough analysis of the stochastic model of an increasing seasonal cycle shows some qualitatively similar results (Fig. 4) to the analysis of the real sea-ice data. In the model, imposed growth in the amplitude in the lower half of the seasonal cycle has been underway for over a decade before the residuals are detected as bi-modal. In contrast, in the real data the increase in amplitude of the seasonal cycle is abrupt (Fig. 1c) and is detected immediately (Fig. 3).
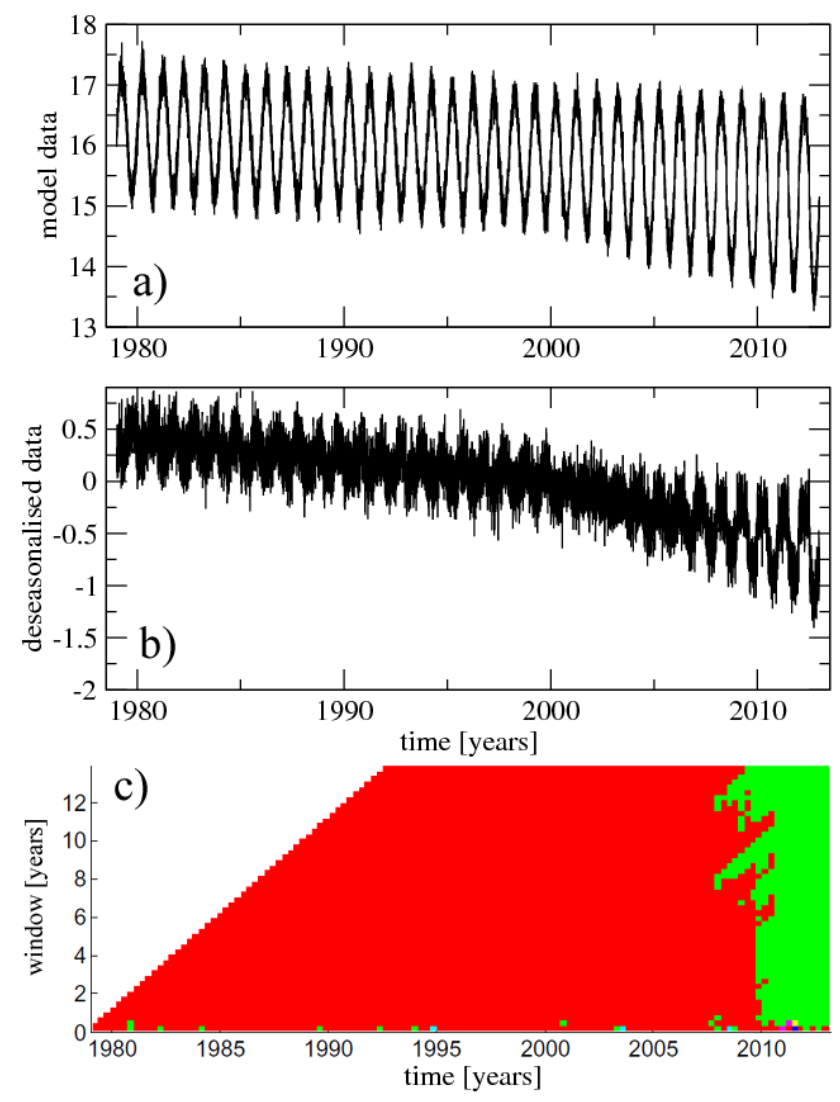

Fig. 4. Model "daily" data with an overall decline and increase in the amplitude of the lower half of the sine wave "seasonal cycle" as described in the text: (a) raw data, (b) deseasonalised data and (c) contour plot of the number of detected states in the deseasonalised data.

\subsection{Early warnings?}

Having hypothesized that a bifurcation may have occurred in Arctic sea-ice cover, we tested this by examining whether it was preceded (or followed) by any signals of destabilization in the form of critical slowing down. However, a caveat here is that the inferred bifurcation (Figs. 3, A2), if correct, represents the creation of a new ice cover attractor (for the residual fluctuations) rather than the total loss of stability of the existing ice cover attractor. Hence the existing ice cover attractor may not show clear destabilization prior to the bifurcation.

Prior to 2007 there is no consistent early warning signal of destabilization (Fig. 5c, e, g). The indicators all increased around the anomalous sea-ice maximum in 1996, but then they all declined toward 2007, consistent with our potential reconstruction (Fig. 3c). The only early warning signal prior to 2007 is a rise in the DFA-indicator in analysis of sea ice extent (Fig. A3). Sensitivity analysis confirms this is the only robust increase across the three indicators and the two datasets, prior to 2007 (Fig. A4). Thus, there was no consistent early warning signal of critical slowing down before the 


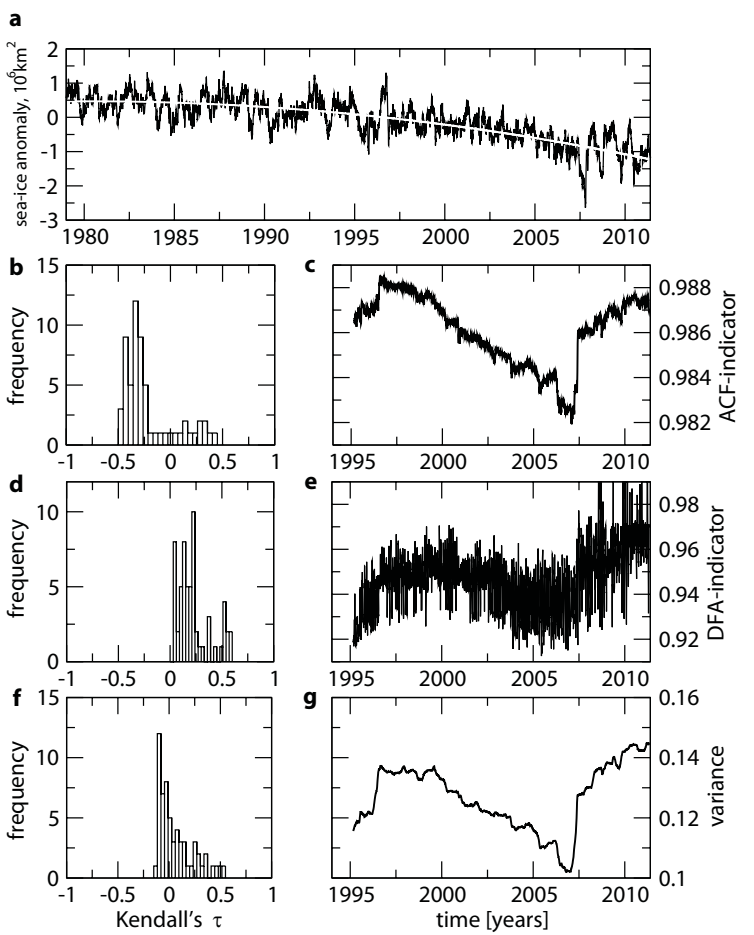

Fig. 5. Search for early warning signals of bifurcation in Arctic sea-ice area data. (a) Sea-ice area anomaly (as in Fig. 2a) showing the quadratic downward trend that is removed prior to calculating the instability indicators. Right panels show example indicators using a sliding window of length half the series, with results plotted at the end of the sliding window. Indicators from (c) autocorrelation function (ACF), (e) detrended fluctuation analysis (DFA) and (g) variance. Left panels show histograms of the Kendall statistic for the trend in the indicators when varying the sliding window length from 1/4 to 3/4 of the series: (b) ACF-indicator; (d) DFA-indicator; (f) variance.

hypothesized bifurcation. Instead the sea ice showed signs of increasing stability in the preceding decade, contrary to what would be expected from an approach to bifurcation.

The sea-ice retreat in 2007 caused abrupt increases in all the indicators, which have continued to rise since then (Fig. 5c, e, g). Sensitivity analysis reveals a robust upward trend in the DFA-indicator across the whole dataset (Fig. 5d), but no robust overall trend in the ACF-indicator or variance (Fig. 5b, f). These results are reproduced in analysis of the shorter record of sea-ice extent data (Fig. A3). The rise in the DFA-indicator could be consistent with the sea ice having increasing "memory" of its earlier states due to critical slowing down (Livina and Lenton, 2007). The somewhat different behaviour of the ACF and DFA indicators could then be explained by the different time scales used for their calculation. The ACF-indicator, based only on lag-1 autocorrelation (here from one day to the next), may be monitoring the behaviour of fast decay modes unrelated to critical slowing down. The DFA-indicator in contrast is calculated on time scales up to
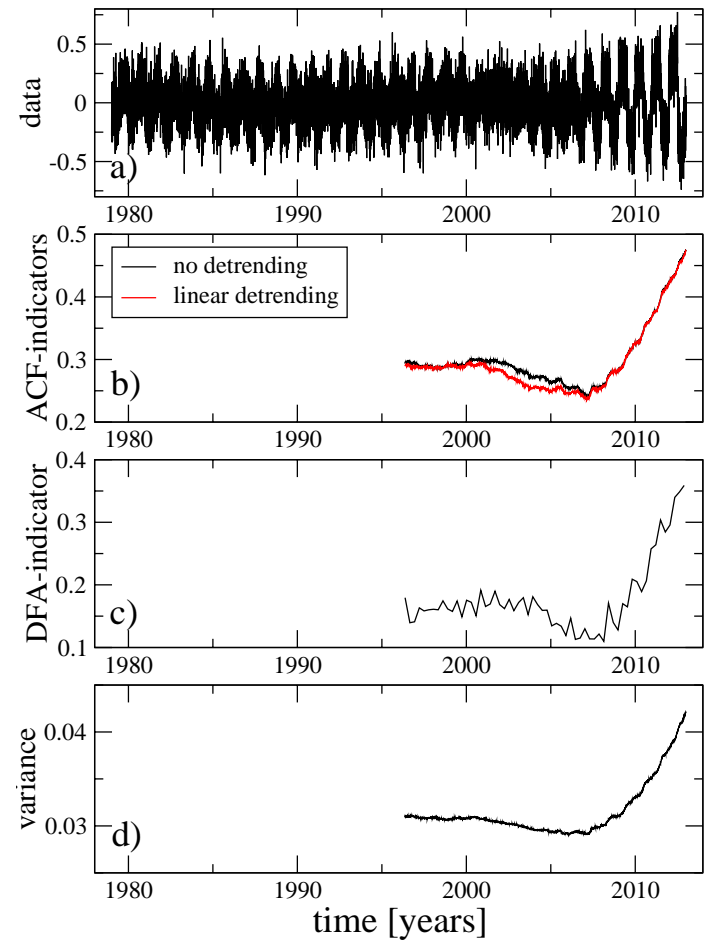

Fig. 6. (a) Detrended deseasonalised model data (Fig. 4) and its early warning indicators: (b) ACF-indicators (with and without linear detrending within sliding windows); (c) DFA-indicator; (d) variance.

100 days, which should be long enough to capture the slowest recovery mode of the sea ice.

We conclude that overall the indicators detect a profound shift in the data in 2007, but do not forewarn of it. This does not convincingly support the bifurcation interpretation. Since 2007 an ongoing destabilization has been detected.

The stochastic model of an increasing seasonal cycle shows no clear trend in the ACF or DFA indicators or the variance, followed by a steady rise in all the indicators as the residual data become bi-modal (Fig. 6). However, there are no abrupt increases in the ACF-indicator of critical slowing down or the variance (Fig. 6), as there are in analysis of the sea-ice data around 2007 (Fig. 5). This is consistent with the change in amplitude of the seasonal cycle being much more abrupt in the real data than in the model.

\subsection{Seasonal analysis}

Our results may be sensitive to the fact that land masses mute variations in winter-spring ice area (Eisenman, 2010), whereas summer-autumn area is less affected. To address this we analysed a derived index of "equivalent sea-ice extent" (Eisenman, 2010), which is based on the latitude of the sea-ice edge where it is free to migrate, converted to an area, assuming there were no continents present. Fluctuations are much larger in this index, and recent summer-autumn ice 
retreats no longer stand out as anomalous (Eisenman, 2010); hence no recent shift from uni-modal to multi-modal residuals is detected (Fig. A5). However, there is still a signal of overall destabilization (Fig. A6), which appears before the signal in actual sea-ice area (Fig. 5). This suggests the abrupt change in the data detected in 2007 could be (at least partly) a geographic property of the shrinkage of summer-autumn ice cover away from the continents facilitating larger fluctuations (Eisenman, 2010).

To examine whether this is the case, we subdivided the original data (Fig. 1a) into two composite series: summer-autumn (June-November inclusive) and winter-spring (December-May inclusive), removing the mean cycle from each, and re-running the analysis. Both subsets of the data carry part of the signal of abrupt change in 2007 (Fig. 7), suggesting the change in the dynamics is not purely a summer-autumn phenomenon. The signal is clearest in summer-autumn, but does not span as wide a range of window lengths as in the full data analysis. However, the summer-autumn data do show upward trends in the ACF and DFA indicators and (less clearly) the variance (Fig. 8), which are generally stronger than in the full dataset (Fig. 5). In contrast, the winter-spring data show no convincing upward trends in any of the indicators (Fig. 9). Thus, the recent signal of increasing auto-correlation and variance (i.e. destabilization) is associated primarily with summer-autumn seaice fluctuations.

\subsection{Summary and geophysical mechanisms}

An abrupt and persistent change in sea-ice dynamics is detected to have occurred in 2007. This involves an extra $\sim 10^{6} \mathrm{~km}^{2}$ or more sea-ice loss each summer-autumn since then. Our initial hypothesis that this abrupt increase in the amplitude of the seasonal cycle of sea-ice variability occurred through a bifurcation mechanism (Livina and Lenton, 2012) is not consistently supported. Thus, the underlying causal mechanism remains uncertain. Still, there must be some amplifying positive feedback mechanisms contributing to the abrupt increase in summer-autumn ice loss.

Statistical models such as ours cannot shed light on these underlying geophysical mechanisms. However, several positive feedbacks have been identified in recent data and are worth mentioning. Sea-ice retreat since 1979 has exposed a dark ocean surface, causing $85 \%$ of the Arctic region to receive an increase in solar heat input at the surface, with an increase of $5 \%$ per year in some regions (Perovich et al., 2007). This is warming the upper Arctic Ocean and contributing to melting on the bottom of the sea ice (Perovich et al., 2008). Sea-ice retreat is also amplifying warming of the lower atmosphere in the Arctic (Screen and Simmonds, 2010), which is shifting precipitation from snow to rainfall, and where rain lands on the remaining sea ice cover, it is encouraging melt (Screen and Simmonds, 2011). The loss of multi-year ice thins the average ice cover making it more vulnerable to
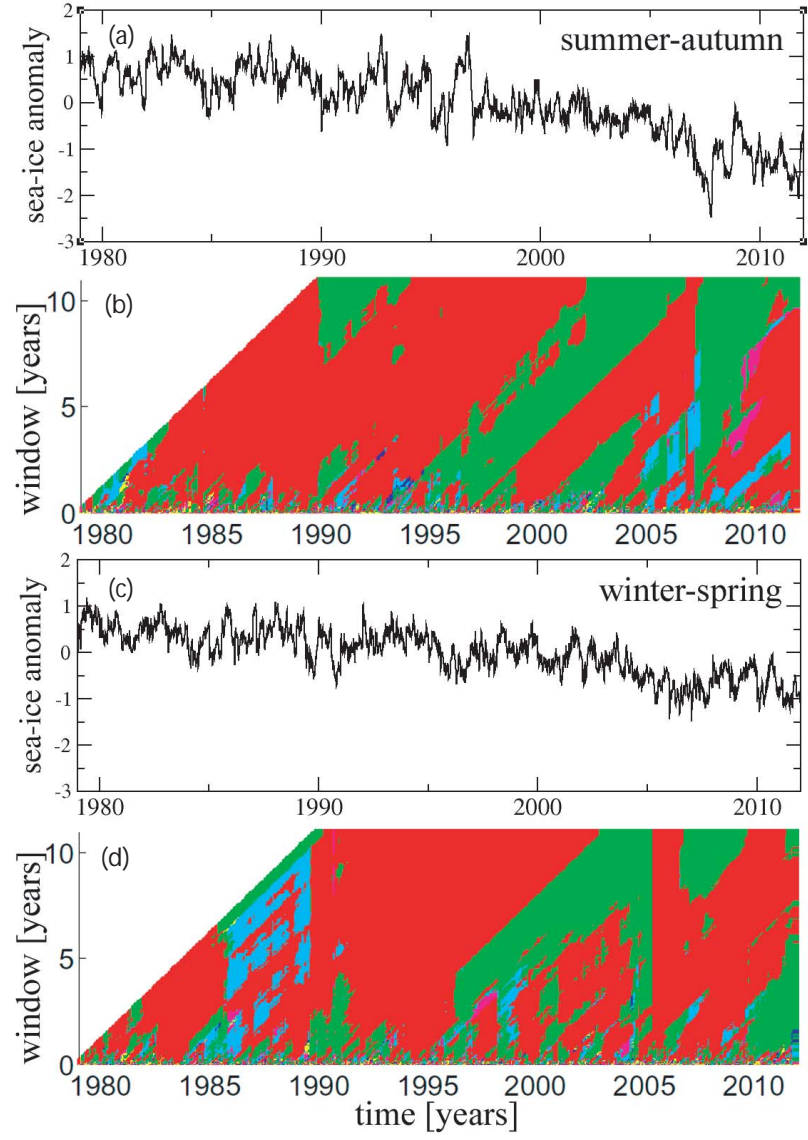

Fig. 7. Potential analysis of summer-autumn and winter-spring Arctic sea-ice area data. (a) Summer-autumn sea-ice area anomaly, daily data with mean cycle removed. (b) Contour plot of number of detected states. (c) Winter-spring sea-ice area anomaly, daily data with mean cycle removed. (d) Contour plot of number of detected states.

further summer losses (Comiso, 2012). Finally, sea-ice loss is beginning to change atmospheric circulation patterns (Overland and Wang, 2010) (although how that feeds back to ice cover is unclear).

The abrupt increase in the seasonal cycle that we detect clearly does not involve total seasonal sea-ice loss and hence is sub-Arctic in scale. However, there may be a precedent for this; past abrupt Arctic cooling and warming events have been linked to switches between alternative states for sea-ice cover in the Barents and Kara seas region (Bengtsson et al., 2004; Semenov et al., 2009). Such sub-Arctic-scale switches can still have significant impacts; indeed recent ice loss from the Barents and Kara seas has been linked to cold winter extremes over Eurasia (Petoukhov and Semenov, 2010). The connection between surface temperature, sea level pressure and winds in the Arctic region, and their effect on the sea-ice cover, is discussed by Comiso (2012). 

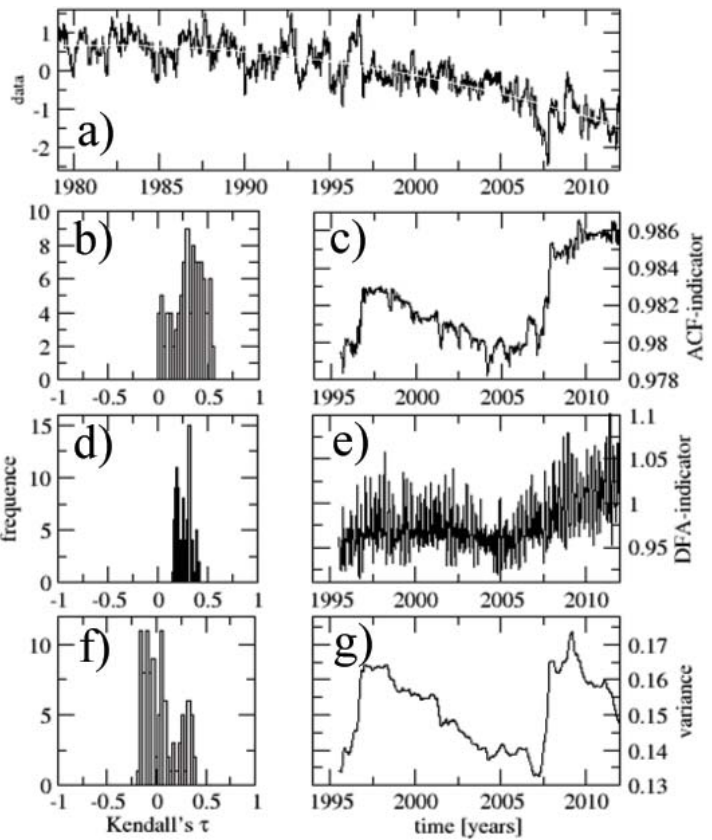

Fig. 8. Search for early warning signals of bifurcation in summer-autumn Arctic sea-ice area. (a) Summer-autumn sea-ice area anomaly (as in Fig. 7a) showing the quadratic downward trend that is removed prior to calculating the instability indicators. Right panels show example indicators from (c) autocorrelation function (ACF), (e) detrended fluctuation analysis (DFA) and (g) variance, results plotted at end of a sliding window of length half the series. Left panels show histograms of the Kendall statistic for the trend in the indicators when varying the sliding window length from 1/4 to 3/4 of the series: (b) ACF-indicator; (d) DFA-indicator; (f) variance.

\section{Conclusions}

We detect an abrupt and persistent increase in the amplitude of seasonal sea-ice variation in 2007. This involves an extra $\sim 10^{6} \mathrm{~km}^{2}$ or more sea-ice loss each summer-autumn then and since. We originally hypothesized that this abrupt change could be explained in terms of a bifurcation in which a new, lower ice cover attractor (for deseasonalised sea-ice fluctuations) appeared and began to be sampled in every summer-autumn from 2007 onwards. However, this interpretation is clearly sensitive to how the seasonal cycle is removed from the raw data, and also to the presence of continental land masses restricting winter-spring ice fluctuations. Furthermore, there was no robust early warning signal of critical slowing down, as would be expected prior to the hypothesized bifurcation. Early warning indicators do however show destabilization of the summer-autumn sea-ice cover since 2007. Overall, the bifurcation hypothesis lacks consistent support. Instead we can say that there has been an abrupt and persistent jump in the amplitude of the seasonal cycle of Arctic sea-ice cover in 2007 (Ditlevsen, 2012), but the underlying causal mechanism remains uncertain. We describe this as a (non-bifurcation) "tipping point", because it involved an
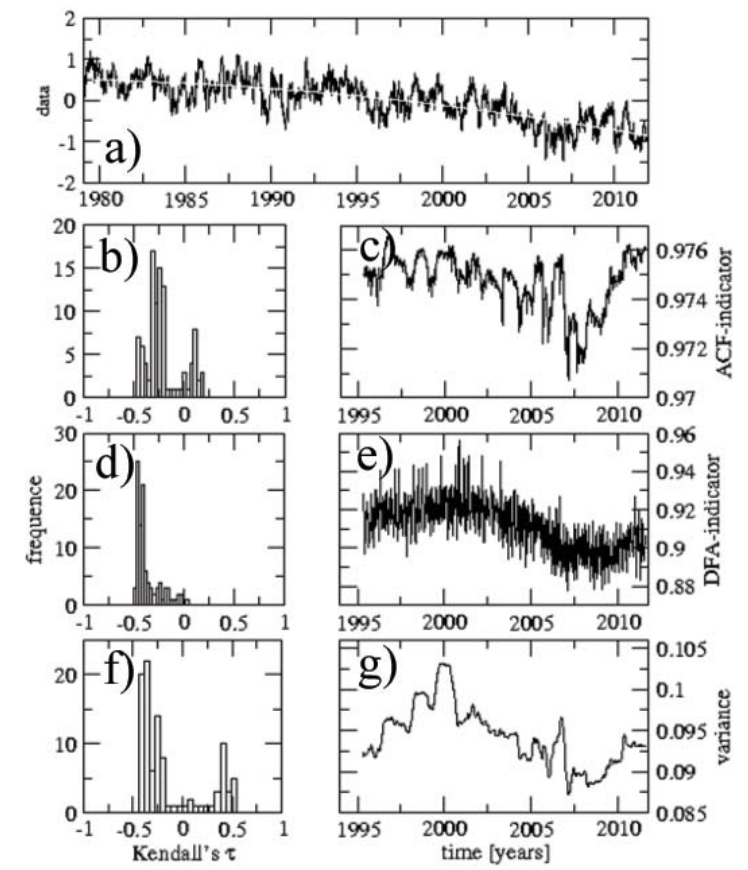

Fig. 9. Search for early warning signals of bifurcation in winterspring Arctic sea-ice area. (a) Winter-spring sea-ice area anomaly (as in Fig. 7c) showing the quadratic downward trend that is removed prior to calculating the instability indicators. Right panels show example indicators from (c) autocorrelation function (ACF), (e) detrended fluctuation analysis (DFA) and (g) variance, results plotted at end of a sliding window of length half the series. Left panels show histograms of the Kendall statistic for the trend in the indicators when varying the sliding window length from 1/4 to $3 / 4$ of the series: (b) ACF-indicator; (d) DFA-indicator; (f) variance.

abrupt, qualitative change in the sea-ice dynamics, without any evidence for a large forcing perturbation; i.e. the abruptness resides in the internal dynamics of the Arctic climate system.

Our statistical methods detected this "tipping point" and its time of onset suggesting they might usefully be applied to real-time analysis of diverse climatological data (albeit in this case the change retrospectively appears fairly clear in the raw data). However, statistical methods cannot shed light on geophysical mechanisms. To make progress on the underlying causal mechanisms requires process-based models. Potentially the statistical indicators of stability could be used to help re-calibrate the sensitivity of process-based models, which have generally proved to be unable to capture the observed abruptness of decline of the Arctic sea-ice cover. 


\section{Appendix A}

\section{Auxiliary materials}
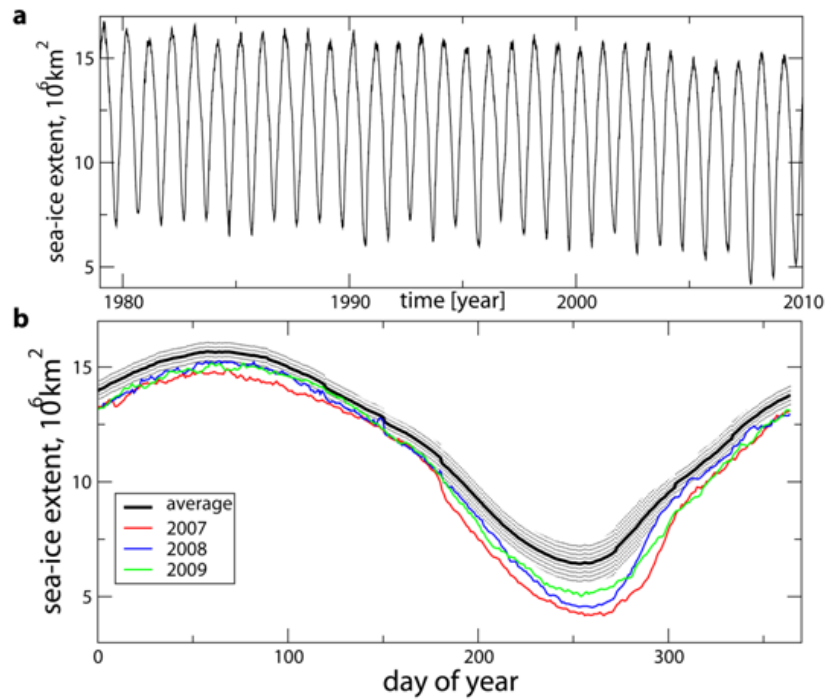

Fig. A1. Arctic sea-ice extent from satellite data. (a) Arctic sea-ice extent from Eisenman (2010) and available at ftp://ftp.agu.org/apend/gl/2010gl043741. (b) The mean annual cycle of the extent data over 1979-2009 (solid line, shaded area denotes $2 \sigma$ error bars), together with the last three anomalous years.
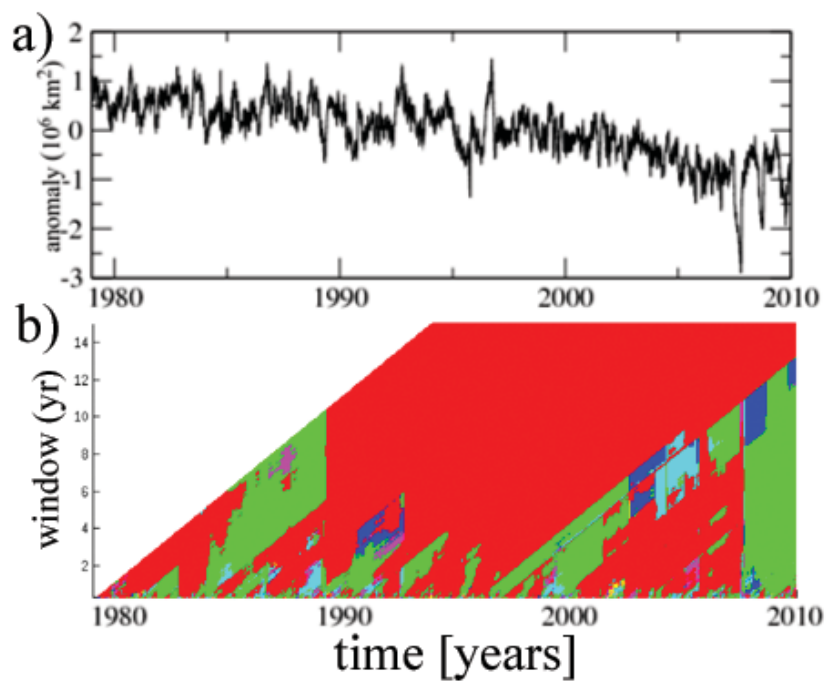

Fig. A2. Analysis of Arctic sea-ice extent. (a) Sea-ice extent anomaly, daily data with mean seasonal cycle removed. (b) Contour plot of number of detected states, where red $=1$ detected state, green $=2$, cyan $=3$, and magenta $=4$. Results plotted as a function of sliding window length at the end of the window.

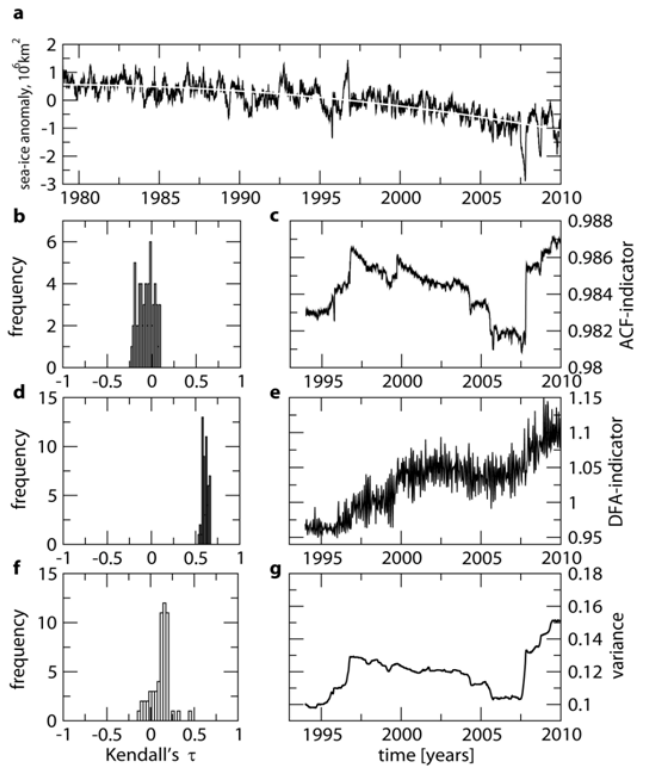

Fig. A3. Search for early warning signals of bifurcation in Arctic sea-ice extent data. (a) Sea-ice extent anomaly (as in Fig. A2a) showing the quadratic downward trend that is removed prior to calculating the instability indicators. Right panels show example indicators from (c) autocorrelation function (ACF), (e) detrended fluctuation analysis (DFA) and (g) variance, results plotted at end of a sliding window of length half the series. Left panels show histograms of the Kendall statistic for the trend in the indicators when varying the sliding window length from 1/4 to 3/4 of the series: (b) ACF-indicator; (d) DFA-indicator; (f) variance.
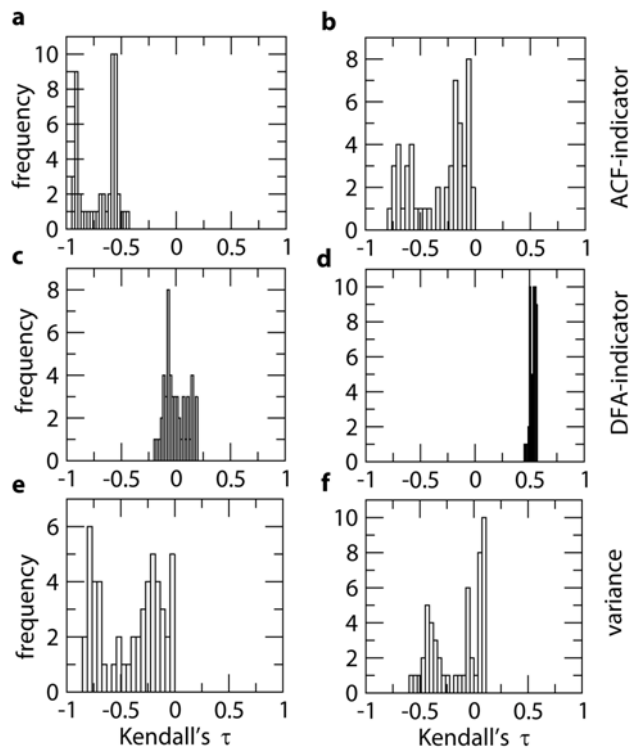

Fig. A4. Destabilisation indicators calculated up to 2007. From (a), (c), (e) sea-ice area anomaly, (b), (d), (f) sea-ice extent anomaly (both after detrending). Sensitivity analysis when varying sliding window length for Kendall trend statistic of (a) and (b) ACFindicator, (c) and (d) DFA-indicator, (e) and (f) variance. 


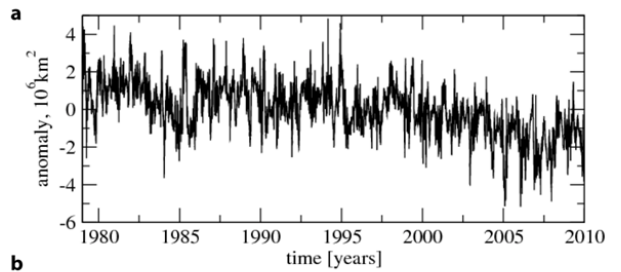

b

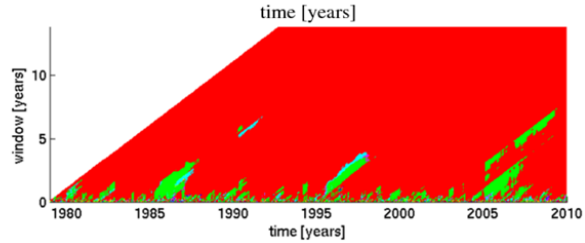

Fig. A5. Potential analysis of equivalent sea-ice extent index. (a) Dataset constructed by Eisenman (2010) and available at ftp: //ftp.agu.org/apend/gl/2010g1043741, which is based on the latitude of the Arctic sea ice edge where the ice is free to migrate, converted to an equivalent area, assuming there were no land masses in the high northern latitudes. (b) Contour plot of number of detected states, where red $=1$ detected state, green $=2$, cyan $=3$, and magenta $=4$. Results plotted as a function of sliding window length at the end of the window. No bifurcation is detected in this dataset, because it has much higher internal variability than sea ice extent (Fig. A3g). Recent observed ice extent anomalies are dwarfed by earlier, larger fluctuations that are inferred to have occurred had the continents not got in the way of winter ice variations.

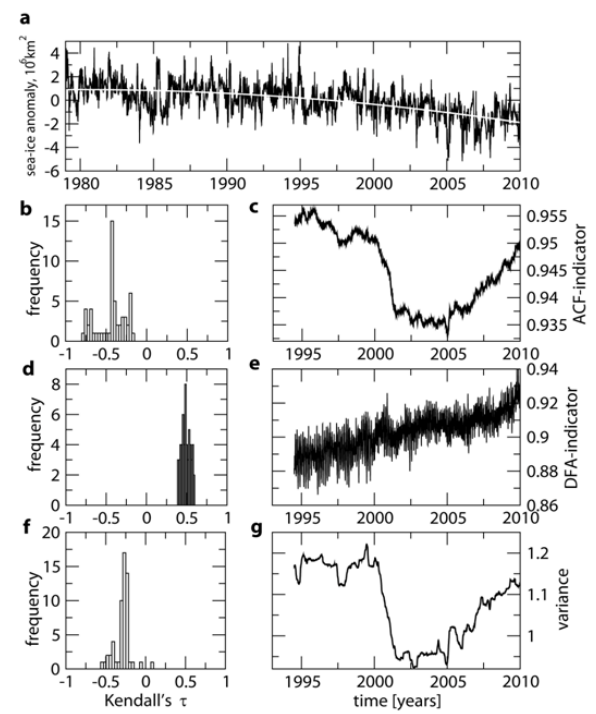

Fig. A6. Search for signals of destabilisation in equivalent sea-ice extent. (a) Equivalent sea-ice extent index (as in Fig. A2) showing the quadratic downward trend that is removed prior to calculating the instability indicators. Right panels show example indicators from (c) autocorrelation function (ACF), (e) detrended fluctuation analysis (DFA) and (g) variance; results are plotted at end of a sliding window of length half the series. Left panels show histograms of the Kendall statistic for the trend in the indicators when varying the sliding window length from 1/4 to 3/4 of the series: (b) ACFindicator; (d) DFA-indicator; (f) variance.
Acknowledgements. The research was supported by the NERC project "Detecting and classifying bifurcations in the climate system" (NE/F005474/1) and by the AXA Research Fund through a postdoctoral fellowship for V. N. L. We thank J. Imbers Quintana and A. Lopes for discussions at the outset of this work, M. Scheffer and P. Ditlevsen for discussions over the interpretation of the results, and two anonymous reviewers for their robust critiques of the Discussion paper. The research was carried out on the High Performance Computing Cluster supported by the Research Computing Service at the University of East Anglia.

Edited by: H. Eicken

\section{References}

Abbot, D. S., Silber, M., and Pierrehumbert, R. T.: Bifurcations leading to summer Arctic sea ice loss, J. Geophys. Res., 116, D19120, doi:10.1029/2011JD015653, 2011.

Amstrup, S. C., Deweaver, E. T., Douglas, D. C., Marcot, B. G., Durner, G. M., Bitz, C. M., and Bailey, D. A.: Greenhouse gas mitigation can reduce sea-ice loss and increase polar bear persistence, Nature, 468, 955-958, 2010.

Bengtsson, L., Semenov, V. A., and Johannessen, O. M.: The Early Twentieth-Century Warming in the Arctic - A Possible Mechanism, J. Climate, 17, 4045-4057, 2004.

Boe, J., Hall, A., and Qu, X.: September sea-ice cover in the Arctic Ocean projected to vanish by 2100, Nat. Geosci., 2, 341-343, 2009.

Cavalieri, D., Parkinson, C., Gloerson, P., and Zwally, H.: Sea ice concentrations from Nimbus-7 SMMR and DMSP SSM/I passive microwave data, 1978-2007, http://nsidc.org/ data/nsidc-0051.html, Natl. Snow and Ice Data Cent., Boulder, Colo., 1996.

Cimatoribus, A. A., Drijfhout, S. S., Livina, V., and van der Schrier, G.: Dansgaard-Oeschger events: tipping points in the climate system, Clim. Past Discuss., 8, 4269-4294, doi:10.5194/cpd-84269-2012, 2012.

Comiso, J.: Large decadal decline of the Arctic multiyear ice cover, J. Climate, 25, 1176-1193, 2012.

Ditlevsen, P. D.: Interactive comment on "A recent bifurcation in Arctic sea-ice cover" by: Livina, V. N. and Lenton, T. M., The Cryosphere Discuss., 6, C1187-C1190, 2012.

Ditlevsen, P. D. and Johnsen, S. J.: Tipping points: Early warning and wishful thinking, Geophys. Res. Lett., 37, L19703, doi:10.1029/2010GL044486, 2010.

Eisenman, I.: Geographic muting of changes in the Arctic sea ice cover, Geophys. Res. Lett., 37, L16501, doi:10.1029/2010GL043741, 2010.

Eisenman, I. and Wettlaufer, J. S.: Nonlinear threshold behavior during the loss of Arctic sea ice, P. Natl. Acad. Sci. USA, 106, 28-32, 2009

Held, H. and Kleinen, T.: Detection of climate system bifurcations by degenerate fingerprinting, Geophys. Res. Lett., 31, L23207, doi:10.1029/2004GL020972, 2004.

Kendall, M. G.: Rank Correlation Methods, Charles Griffin \& Company Limited, London, 1948.

Lenton, T. M.: Early warning of climate tipping points, Nature Climate Change, 1, 201-209, 2011. 
Lenton, T. M., Held, H., Kriegler, E., Hall, J. W., Lucht, W., Rahmstorf, S., and Schellnhuber, H. J.: Tipping Elements in the Earth's Climate System, P. Natl. Acad. Sci. USA, 105, 1786-1793, 2008.

Lindsay, R. W. and Zhang, J.: The Thinning of Arctic Sea Ice, 19882003: Have We Passed a Tipping Point?, J. Climate, 18, 48794894, 2005.

Livina, V. N. and Lenton, T. M.: A modified method for detecting incipient bifurcations in a dynamical system, Geophys. Res. Lett., 34, L03712, doi:10.1029/2006GL028672, 2007.

Livina, V. N. and Lenton, T. M.: A recent bifurcation in Arctic sea-ice cover, The Cryosphere Discuss., 6, 2621-2651, doi:10.5194/tcd-6-2621-2012, 2012.

Livina, V. N., Kwasniok, F., and Lenton, T. M.: Potential analysis reveals changing number of climate states during the last $60 \mathrm{kyr}$, Clim. Past, 6, 77-82, doi:10.5194/cp-6-77-2010, 2010.

Livina, V. N., Kwasniok, F., Lohmann, G., Kantelhardt, J. W., and Lenton, T. M.: Changing climate states and stability: from Pliocene to present, Clim. Dynam., 37, 2437-2453, 2011.

Livina, V. N., Ditlevsen, P. D., and Lenton, T. M.: An independent test of methods of detecting and anticipating bifurcations in time-series data, Physica A, 391, 485-496, doi:10.1016/j.physa.2011.08.025, 2012.

Maslanik, J. and J. Stroeve: Near real-time DMSP SSM/I daily polar gridded sea ice concentrations, http://nsidc.org/data/nsidc-0081. html, Natl. Snow and Ice Data Cent., Boulder, Colo., 1999.

Meier, W., Fetterer, F., Knowles, K., Savoie, M., and Brodzik, M. J.: Sea ice concentrations from Nimbus-7 SMMR and DMSP SSM/I passive microwave data, January-December 2008, http://nsidc.org/data/nsidc-0051.html, Natl. Snow and Ice Data Cent., Boulder, Colo., 2006.

Nghiem, S. V., Rigor, I. G., Perovich, D. K., Clemente-Colón, P., Weatherly, J. W., and Neumann, G.: Rapid reduction of Arctic perennial sea ice. Geophys. Res. Lett., 34, L19504, doi:10.1029/2007GL031138, 2007.

Notz, D.: The future of ice sheets and sea ice: Between reversible retreat and unstoppable loss, P. Natl. Acad. Sci. USA, 106, 20590 20595, 2009.

Overland, J. E. and Wang, M.: Large-scale atmospheric circulation changes are associated with the recent loss of Arctic sea ice, Tellus A, 62, 1-9, 2010.
Perovich, D. K. Light, B., Eicken, H., Jones, K. F., Runciman, K., and Nghiem, S. V.: Increasing solar heating of the Arctic Ocean and adjacent seas, 1979-2005: Attribution and role in the ice-albedo feedback, Geophys. Res. Lett., 34, L19505, doi:10.1029/2007GL031480, 2007.

Perovich D. K., J. A. Richter-Menge, K. F. Jones, and Light, B.: Sunlight, water, and ice: Extreme Arctic sea ice melt during the summer of 2007, Geophys. Res. Lett., 35, L11501, doi:10.1029/2008GL034007, 2008

Petoukhov, V. and Semenov, V. A.: A link between reduced BarentsKara sea ice and cold winter extremes over northern continents, J. Geophys. Res., 115, doi:10.1029/2009JD013568, D21111, 2010.

Scheffer, M. Bascompte, J., Brock, W. A., Brovkin, V., Carpenter, S. R., Dakos, V., Held, H., van Nes, E. H., Rietkerk, M., and Sugihara, G.: Early warning signals for critical transitions, Nature, 461, 53-59, 2009.

Screen, J. A. and Simmonds, I.: The central role of diminishing sea ice in recent Arctic temperature amplification, Nature, 464, 1334-1337, 2010.

Screen, J. and Simmonds, I.: Declining summer snowfall in the Arctic: causes, impacts and feedbacks, Clim. Dynam., 38, 2243 2256, doi:0.1007/s00382-011-1105-2, 2011.

Semenov, V. A., Park, W., and Latif, M.: Barents Sea inflow shutdown: A new mechanism for rapid climate changes, Geophys. Res. Lett., 36, L14709, doi:10.1029/2009GL038911, 2009.

Stroeve, J., Holland, M. M., Meier, W., Scambos, T., and Serreze, M.: Arctic sea ice decline: Faster than forecast, Geophys. Res. Lett., 34, L09501, doi:10.1029/2007GL029703, 2007.

Stroeve, J. C., Kattsov, V., Barrett, A., Serreze, M., Pavlova, T., Holland, M., and Meier, W. N.: Trends in Arctic sea ice extent from CMIP5, CMIP3 and observations, Geophys. Res. Lett., 39, L16502, doi:10.1029/2012GL052676, 2012.

Tietsche, S., Notz, D., Jungclaus, J. H., and Marotzke, J.: Recovery mechanisms of Arctic summer sea ice, Geophys. Res. Lett., 38, L02707, doi:10.1029/2010GL045698, 2011.

Winton, M.: Does the Arctic sea ice have a tipping point?, Geophys. Res. Lett., 33, L23504, doi:10.1029/2006GL028017, 2006. 